\title{
Biomarcadores de sensibilização primária e reatividade cruzada em doentes alérgicos a veneno de himenópteros
}

\author{
Biomarkers of primary sensitization and cross-reactivity \\ in patients allergic to Hymenoptera venom
}

Data de receção / Received in: 15/09/2017 Data de aceitação / Accepted for publication in: 05/10/2018

\begin{abstract}
Rev Port Imunoalergologia 20I9;27 (2): I05-II 4
\end{abstract}
Letícia Pestana ${ }^{1,2}$, Alcinda Campos Melo', Elisa Pedro², Manuel Pereira Barbosa ${ }^{2,3}$, Maria Conceição Pereira Santos ${ }^{1,3}$

' Laboratório de Imunologia Clínica, Faculdade de Medicina - Instituto de Medicina Molecular, Universidade de Lisboa

${ }^{2}$ Serviço de Imunoalergologia, Hospital de Santa Maria, CHLN

${ }^{3}$ Clínica Universitária de Imunoalergologia, Faculdade de Medicina, Universidade de Lisboa

\section{RESUMO}

Introdução: A maioria dos doentes com alergia à picada de himenópteros apresenta dupla positividade (testes cutâneos-TC/lgE específica-slgE) para veneno de abelha (A), vespa $(\mathrm{V})$ e Polistes $(\mathrm{P})$, constituindo um problema quer no diagnóstico quer na seleção para imunoterapia específica (IT). Objetivo: Caraterização do perfil de sensibilização de doentes alérgicos à picada de himenópteros, através da utilização de alergénios recombinantes, fosfolipases, hialuronidases e determinantes dos carboidratos para distinguir dupla sensibilização de reatividade cruzada. Avaliação da contribuição do teste de ativação de basófilos (TAB) no diagnóstico. Métodos: Foram incluídos 124 doentes com história de reação sistémica (RS), não submetidos a IT, e um grupo-controlo de 30 indivíduos sem história de reação. Os TC foram realizados com extratos comerciais (EC) de veneno, assim como determinação de slgE sérica. Doseou-se também slgE para recombinantes, Api ml, Ves v5, Ves vl, Pol d5 e CCD. Em doentes com TC/slgE negativos, foi realizado o TAB em seis concentrações. Resultados: Baseada na slgE, 66 doentes (53\%) encontravam-se polissensibilizados e 38 doentes (31\%) monossensibilizados. Em 20 doentes (16\%) os TC/slgE foram negativos. Dos 66 doentes polissensibilizados, 32 (49\%) apresentavam dupla sensibilização, 23 (35\%) reatividade cruzada e II (16\%) slgE negativa para recombinantes. A slgE para CCD foi positiva em 25 doentes, todos com dupla sensibilização com EC, demonstrando reatividade cruzada em 23. Os 20 doentes com TC/slgE negativos apresentaram slgE negativa para os recombinantes. A especificidade para os quatro componentes moleculares foi elevada. O TAB foi realizado em 5 doentes, sendo posi- 
tivo em 3 para veneno (A). Conclusão: Os alergénios recombinantes para veneno $(A)$ e $(V)$ complementam o diagnóstico de alergia à picada de himenópteros, permitindo distinguir dupla sensibilização de reatividade cruzada e uma melhor seleção de doentes para IT. O TAB poderá ser um contributo importante em casos de difícil diagnóstico.

Palavras-chave: Alergia a veneno de himenópteros, alergénios recombinantes, dupla sensibilização, reatividade cruzada, teste de ativação de basófilos.

\section{ABSTRACT}

Background: Diagnostic tests in patients allergic to hymenoptera venom are often positive for bee (B), wasp (W) and Polistes $(P)$ venom, being difficult to choose the appropriate immunotherapy. Aim: Characterization of sensitization profile of patients allergic to hymenoptera venom using recombinant allergens, to distinguish true double sensitization from crossreactivity of venoms. Evaluation of basophil activation test (BAT) contribution in diagnosis of patients with systemic reactions with negative skin test (ST) and specific lgE (slgE). Methods: 124 patients with systemic reactions to Hymenoptera stings and 30 controls without history of reaction to insect stings were included. ST were performed with commercial extracts (CE) of (B), (W) and (P) venoms, as well as slgE with the same extracts and recombinant allergens for Api $m l$, Ves $v 5$ and Ves $v l$, and to Pol $d 5$ and carbohydrate determinants (CCD). In patients with negative ST/s/gE, BAT was performed in six concentrations using both venoms. Results: Based on slgE, 66 patients (53\%) were polisensitized and 38 patients (31\%) were monosensitized. In 20 patients (16\%) ST and slgE were negative. Of the 66 polisensitized patients, 32 (49\%) had double sensitization, 23 (35\%) revealed cross-reactivity and II (16\%) had none recombinants allergens. slgE to CCD was positive in 25 patients, all with double sensitization with CE, showing crossreactivity between species in 23 . The 20 patients with negative ST/slgE had also negative allergen recombinants. The specificity for the four components was high, since only a control had positive slgE to Vesvl/ Vesv5. BAT was performed in 5 patients to both venoms and it was positive in 3 for B venom. Conclusions: Recombinant allergens for bee and wasp venom slgE complements the diagnosis of venom allergy, allowing discrimination between true double sensitization and crossreactivity, as well as a better selection of patients for VIT. BAT seems to be useful in cases of difficult diagnosis.

Keywords: Hymenoptera venom allergy, recombinant allergens, double sensitization, cross-reactivity, basophil activation test.

\section{INTRODUÇÃO}

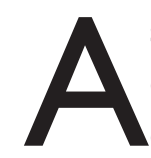
s picadas de insetos desencadeiam, na maioria dos casos, reações locais devido à toxicidade do veneno. No entanto, está descrito que cerca de 3,5\% da população em geral tem história de reação sistémica (RS), incluindo anfilaxia'. A imunoterapia (IT) é considerada um tratamento altamente eficaz na prevenção de reações graves em doentes alérgicos ao veneno de himenópteros, mas requer um diagnóstico cuidadoso que visa identificar não só a alergia mas também ○ inseto responsável ${ }^{1,2}$. Porém, o diagnóstico, que se baseia na história clínica, testes cutâneos (TC) e na quantificação de anticorpos IgE específicas séricas 
( $\lg \mathrm{E}$ esp) para veneno de himenópteros, pode ser dificultado por vários fatores.

Estima-se que cerca de $50 \%$ dos doentes com alergia ao veneno destes insetos apresentem resultados positivos, TC e/ou IgE esp, tanto para veneno de abelha (Apis mellifera) como para veneno de vespa (Vespula germanica e Polistes dominula, as duas principais espécies no nosso país) $)^{1,2}$. Por outro lado, alguns autores descrevem que mais de $30 \%$ dos doentes com RS à picada de insetos apresentam TC e IgE esp negativos, provavelmente devido à sensibilidade limitada destes métodos ${ }^{3}$. A dupla positividade para veneno de abelha e veneno de vespa pode representar uma verdadeira dupla sensibilização ou reatividade cruzada a ambos os venenos devido à homologia de sequência entre proteínas destes venenos, tais como hialuronidases ou mesmo determinantes dos carboidratos (CCD). ${ }^{4}$ Por outro lado, este diagnóstico serológico pode também ser dificultado pela presença e reconhecimento de fontes alergénicas não relacionadas, como pólen e materiais derivados de plantas (por exemplo, látex), que apesar de não demonstrarem atividade alergénica podem dar origem a falsos positivos ${ }^{5}$. Ainda, nestes casos, pode ser difícil (e às vezes impossível) o doente identificar o inseto responsável pela reação ${ }^{6}$. $O$ diagnóstico assertivo é crucial na seleção de venenos para IT, uma vez que o tratamento com ambos os venenos está apenas indicado nos casos de verdadeira dupla sensibilização.

Durante um longo período de tempo, apenas os testes laboratoriais por inibição de anticorpos lgE permitiam a distinção entre dupla sensibilização primária e reatividade cruzada. Estes testes são dispendiosos, demorados, difíceis de interpretar e, portanto, raramente utilizados na prática clínica ${ }^{6-10}$. O desenvolvimento contínuo de testes in vitro mais específicos e sensíveis tem-se revelado muito importante. Como tem sido amplamente demonstrado em diversas áreas, os alergénios recombinantes podem ser ferramentas úteis no diagnóstico de alergia ao veneno de himenópteros, tornando-o mais preciso, sendo a sua utilidade, no diagnóstico in vitro e in vivo, já demonstrada em vários estudos $7,9,10$.
Neste trabalho, os autores avaliam a utilidade dos alergénios recombinantes: fosfolipase $A 2$ de veneno de abelha (Api $\mathrm{ml}$ ), fosfolipase Al, antigénio 5 (Ves vl /Ves $\checkmark 5)$, Pol d5 de veneno de vespa e CCD, no diagnóstico de alergia a veneno de himenópteros, identificando dupla sensibilização primária e reatividade cruzada. Por outro lado, avaliou-se a contribuição do teste de ativação de basófilos (TAB) com o veneno de abelha e vespa no diagnóstico de doentes com história de RS e com TC e lgE esp negativos para ambos os venenos.

\section{MÉTODOS}

\section{População}

Neste estudo foram incluídos 124 doentes (76 homens, 48 mulheres, idades compreendidas entre os $21 \mathrm{e}$ os 78 anos, idade média $52 \pm 3,8 \mathrm{I}$ ) com história de RS a picadas de himenópteros, não submetidos a IT. Os critérios de inclusão foram no mínimo uma RS de tipo imediato de grau I a IV (de acordo com a classificação Muller'l) após picada de himenópteros e lgE esp positiva para veneno destes insetos.

O grupo-controlo incluiu 30 indivíduos, não atópicos, sem história de reação à picada de himenópteros, com IgE esp negativa para veneno de abelha, veneno de vespa e veneno de polistes. O projeto foi aprovado pela Comissão de Ética do Hospital de Santa Maria/Faculdade de Medicina da Universidade de Lisboa. Todos os indivíduos assinaram o consentimento informado para a colheita e o processamento das amostras.

\section{Testes cutâneos}

Foram realizados testes cutâneos intradérmicos com veneno de abelha, veneno de vespa e veneno de polistes (Bial Aristegui, Bilbao, Espanha) em todos os doentes com concentrações sucessivas de 0,000I, 0,00I, 0,0I e $0,1 \mathrm{mg} / \mathrm{mL}$ com um controlo positivo (dicloridrato de histamina) e controlo negativo (solução salina), de acordo com as recomendações da European Academy of Allergo- 
logy and Clinical Immunology ${ }^{8}$. Um diâmetro de pápula $\geq 3$ $\mathrm{mm}$ foi considerado como uma reação positiva.

\section{Quantificação de IgE sérica}

A determinação da IgE específica sérica (IgE esp) para os extratos convencionais de veneno de abelha, vespa, veneno de polistes, determinantes dos carboidratos CCD (MUXF3), alergénios recombinantes major fosfolipase A2 (Api m I), fosfolipase Al (Ves v I) e antígeno 5 (Ves $\vee 5$ ) foi avaliada no soro de doentes e grupo-controlo de acordo com as instruções do fabricante, ImmunoCAP (Thermo Fisher Diagnostics, Upsala, Suécia). Uma concentração $\geq 0,35 \mathrm{kU} / \mathrm{L}$ foi definida como teste positivo.

\section{Teste de ativação dos basófilos}

O TAB foi realizado com o extrato estandardizado de veneno de abelha e vespa nas concentrações de 0,02 ; 0,$04 ; 0,2 ; 1 ; 2,5 ; 5 \mu g / m l$, de acordo com a literatura, usando o método Flow2 CAST (Bühlmann Laboratories AG, Schönenbuch, Suíça) e com as instruções do fabricante. Resumidamente, a $50 \mu \mathrm{l}$ de sangue total heparinizado adicionaram-se $50 \mu \mathrm{l}$ de cada uma das concentrações dos venenos, $50 \mu \mathrm{l}$ do péptido quimiotático $\mathrm{N}$-formil-Met-Leu-Phee (FMLP) e um anticorpo monoclonal anti-FcERI (controlos positivos) e $50 \mu \mathrm{l}$ de solução tampão-PBS (controlo negativo). A cada tubo foram adicionados $100 \mu \mathrm{l}$ de tampão de estimulação (IL-3), $20 \mu \mathrm{l}$ dos anticorpos monoclonais, anti-CD63-FITC e anti-CCR3-PE seguindo-se uma incubação de 15 minutos a $37^{\circ} \mathrm{C}$. Foi posteriormente efetuada a lise dos eritrócitos, lavagem e ressuspensão das células em PBS. A aquisição por citometria de fluxo (FACSCalibur, Becton Dickinson) ocorreu nas duas horas a seguir à finalização da técnica. A população de basófilos foi identificada como $\mathrm{CCR}^{+} \mathrm{e}$ a ativação foi determinada pela expressão de CD63, sendo analisadas usando o software Flow Jo (TreeStar). Em cada ensaio, pelo menos 800 basófilos foram avaliados. Os resultados foram considerados positivos quando se verificou uma percentagem de ativação superior a $10 \%$ com índice de estimulação igual ou superior a dois como confirmado em estudos anteriores ${ }^{12,13,14}$.

\section{Análise Estatística}

A análise estatística foi realizada usando o programa SPSS para software Windows (SPSS Inc., Chicago, EUA).

\section{RESULTADOS}

Doze doentes não identificaram o tipo de inseto em pelo menos um dos episódios, 56 doentes referiram picada por abelha, 38 por vespa e 18 por ambos os insetos. Cinquenta e cinco doentes apresentaram TC positivo para mais de um veneno e 47 apenas para um veneno. Vinte doentes descreveram uma RS para veneno de abeIha e/ou veneno de vespa, mas verificaram-se TC e lgE esp negativos para ambos os venenos.

Os resultados obtidos da avaliação da lgE esp para extrato de veneno convencional (tendo sido este o critério utilizado para definir mono/polissensibilização) demonstraram monossensibilização em 38 doentes (31\%) (abelha, $n=31$; vespa, $n=4$; polistes $n=3$ ), polissensibilização em 66 (53\%) e duplamente negativos em 20 (16\%).

Nos doentes monossensibilizados a veneno de abelha, a IgE esp para o recombinante Api $m$ I foi detetada em 2 | dos 3 I doentes com alergia a veneno de abelha, revelando uma sensibilidade de $68 \%$. Por outro lado, a lgE esp para Ves v I foi detetada em todos os doentes com alergia a veneno de vespa (sensibilidade de 100\%) e a Pol d5 foi apenas detetada num doente do grupo dos monossensibilizados a veneno de polistes, sendo a sua sensibilidade de $33 \%$.

Nenhum dos 7 doentes com alergia a veneno de vespa ou veneno de polistes reagiram a Api m I, correspondendo a uma especificidade de $100 \%$. O mesmo ocorreu nos doentes monossensibilizados a veneno de abelha os quais não revelaram positividade a Ves v I/Ves v 5 . Apenas um controlo positivo apresentou uma lgE esp positiva a Ves $\vee 5$, revelando uma especificidade de $98 \%$ para este componente molecular (Quadro I). 
Quadro I. Caracterização de doentes monossensibilizados com reação sistémica à picada de himenópteros e grupo-controlo

\begin{tabular}{|l|c|c|c|c|}
\hline \multicolumn{1}{|c|}{ ImmunoCAP } & $\begin{array}{c}\text { Positivo apenas a VA } \\
\mathbf{N}=\mathbf{3} \text { I }\end{array}$ & $\begin{array}{c}\text { Positivo apenas a VV } \\
\mathbf{N}=\mathbf{4}\end{array}$ & $\begin{array}{c}\text { Positivo apenas a VP } \\
\mathbf{N}=\mathbf{3}\end{array}$ & $\begin{array}{c}\text { Controlos } \\
\mathbf{N}=\mathbf{3 0}\end{array}$ \\
\hline Positivo apenas a Api mI & $\mathrm{N}=2$ I (68\%) & $\mathrm{N}=0$ & $\mathrm{~N}=0$ & $\mathrm{~N}=0$ \\
\hline Positivo apenas a Ves vI/Ves v 5 & $\mathrm{~N}=0$ & $\mathrm{~N}=4(100 \%)$ & $\mathrm{N}=0$ \\
\hline Positivo apenas a Pol d 5 & $\mathrm{N}=0$ & $\mathrm{~N}=0$ & $\mathrm{~N}=\mathrm{I}(33 \%)$ & $\mathrm{N}=0$ \\
\hline Recombinantes negativos & $\mathrm{N}=10(32 \%)$ & $\mathrm{N}=0$ & $\mathrm{~N}=2(67 \%)$ & $\mathrm{N}=29(97 \%)$ \\
\hline Positivo (CCD) & $\mathrm{N}=0$ & $\mathrm{~N}=0$ & $\mathrm{~N}=7(33 \%)$ & $\mathrm{N}=0$ \\
\hline
\end{tabular}

Anticorpos IgE esp para extrato total e alergénios recombinantes.

VA - veneno abelha; VV - veneno vespa; VP - veneno polistes; CCD - determinantes dos carboidratos.

No grupo dos 66 doentes polissensibilizados, através da avaliação da lgE específica para extrato de veneno convencional, 32 (49\%) apresentaram dupla sensibilização aquando da avaliação com componentes moleculares (lgE esp positiva para Api ml, Ves vl/Pol d5 (I), Api ml/Ves vl (7) ou Api ml/Pol d5 (8); Ves vl/
Pol d5 (9); Ves v5/Pol d5 (2), Ves vl/Ves v5/Pol d5 (5)); 23 (35\%) revelaram reatividade cruzada Api m I (I0); Ves v I (8), Ves v 5 (I), Ves v I/Ves v 5 (2) e Pol d5 (2). Os restantes II doentes (16\%) apresentaram resultados de lgE esp negativos para todos os componentes moleculares.

Quadro 2. Caracterização de doentes polissensibilizados com reação sistémica à picada de himenópteros e grupo-controlo

\begin{tabular}{|c|c|c|c|c|c|}
\hline ImmunoCAP & $\begin{array}{c}\text { Positivo } \\
\text { a VA e VV } \\
N=9\end{array}$ & $\begin{array}{c}\text { Positivo } \\
\text { a VA e VP } \\
N=6\end{array}$ & $\begin{array}{c}\text { Positivo } \\
\text { a VV/VP } \\
\mathbf{N}=21\end{array}$ & $\begin{array}{c}\text { Positivo } \\
\text { a VA/VV e PV } \\
\mathbf{N}=30\end{array}$ & $\begin{array}{l}\text { Controlos } \\
\qquad \mathbf{N}=30\end{array}$ \\
\hline Positivo a Api ml, Ves vl, & $\mathrm{N}=4(44 \%)$ & $N=0$ & $N=0$ & $\mathrm{~N}=3(10 \%)$ & $N=0$ \\
\hline Positivo a Api ml,Pol d 5 & $N=5(56 \%)$ & $N=0$ & $N=0$ & $\mathrm{~N}=3(10 \%)$ & $N=0$ \\
\hline Positivo a Api ml, Ves vl/Pol d 5 & $N=0$ & $N=I(17 \% 9$ & $N=0$ & $N=0$ & $N=0$ \\
\hline Positivo a Ves vl/Ves v 5 & $N=0$ & $N=2(32 \%)$ & $N=0$ & $N=0$ & $N=0$ \\
\hline Positivo a Ves vl/Pol d5 & $N=0$ & $\mathrm{~N}=0$ & $N=7(33 \%)$ & $N=2(7 \%)$ & $N=0$ \\
\hline Positivo a Ves v5/Pol d 5 & $N=0$ & $N=0$ & $\mathrm{~N}=2(10 \%)$ & $N=0$ & $N=0$ \\
\hline Positivo a Ves vl/Ves v5/Pol d 5 & $N=0$ & $N=0$ & $N=5(24 \%)$ & $N=0$ & $N=0$ \\
\hline Positivo só a Api ml & $N=0$ & $N=I(I 7 \%)$ & $N=0$ & $N=9(30 \%)$ & $N=0$ \\
\hline Positivo só a Ves v I & $N=0$ & $N=I(I 7 \%)$ & $N=6(29 \%)$ & $N=I(3 \%)$ & $N=0$ \\
\hline Positivo só a Ves v 5 & $N=0$ & $N=0$ & $N=0$ & $N=I(3 \%)$ & $N=I(3 \%)$ \\
\hline Positivo só a Pol d 5 & $N=0$ & $N=I(I 7 \%)$ & $N=I(5 \%)$ & $N=0$ & $N=0$ \\
\hline Todos recombinantes negativos & $N=0$ & $N=0$ & $N=0$ & $N=I \mid(37 \%)$ & $N=29(97 \%)$ \\
\hline Positivo a CCD & $N=I(I I \%)$ & $N=5(83 \%)$ & $N=9(43 \%)$ & $N=10(33 \%)$ & $N=0$ \\
\hline
\end{tabular}

Anticorpos IgE esp para extrato total e alergénios recombinantes.

$\mathrm{VA}$ - veneno abelha; VV - veneno Vespa; VP - veneno polistes; CCD - determinantes dos carboidratos 
Letícia Pestana, Alcinda Campos Melo, Elisa Pedro, Manuel Pereira Barbosa, Maria Conceição Pereira Santos

Quadro 3. Ativação de basófilos em cinco doentes com história de reação sistémica à picada de insectos e testes cutâneos e lgE específica negativos para veneno de abelha e vespa

\begin{tabular}{|c|c|c|c|c|c|c|c|c|}
\hline Concentração & $\begin{array}{l}\text { Controlo } \\
\text { negativo }\end{array}$ & $\begin{array}{l}\text { Controlo } \\
\text { positivo }\end{array}$ & $\begin{array}{l}0,0 \text { l ug/ml } \\
\text { VA/VV }\end{array}$ & $\begin{array}{l}0,04 \text { ug/ml } \\
\text { VA/VV }\end{array}$ & $\begin{array}{l}0,2 \text { ug/ml } \\
\text { VA/VV }\end{array}$ & $\begin{array}{l}\text { lug/ml } \\
\text { VA/VV }\end{array}$ & $\begin{array}{l}2,5 \mathrm{ug} / \mathrm{ml} \\
\text { VA/VV }\end{array}$ & $\begin{array}{l}\text { 5ug/ml } \\
\text { VA/VV }\end{array}$ \\
\hline Doente I & ।,83 & 22,18 & 2,$29 ; 2,05$ & I,I9;I,23 & I,I 2;।,03 & 5,$13 ; 3,28$ & 10,$57 ; 2,02$ & ।,38; |,42 \\
\hline Doente 2 & I,67 & 11,86 & I,90;1,73 & 2,$48 ; 2,36$ & I,84;1,92 & I,69;2,02 & I I , 50; I,23 & 9,$85 ; 2,25$ \\
\hline Doente 3 & ।,79 & 33,46 & I,39;1,26 & I, I7;1,23 & 2,$38 ; 1,96$ & 5,$43 ; 2,96$ & 15,$20 ; 1,39$ & 8,$09 ; 2,21$ \\
\hline Doente 4 & 2,07 & 33,30 & I,47;।,38 & 0,$94 ; 0,78$ & 0,$87 ; 0,85$ & I,34;।,25 & I,0I;।,34 & I,02; I,06 \\
\hline Doente 5 & 1,43 & 18,18 & I,I0;1,05 & I,52;1,57 & 2,$01 ; 2,04$ & I,45;I,38 & 0,$21 ; 1,15$ & 0,$46 ; 0,26$ \\
\hline
\end{tabular}

Um valor de ativação $\geq 10 \%$ e IE $\geq 2$ foi considerado positivo. VA - veneno abelha; VV - veneno vespa

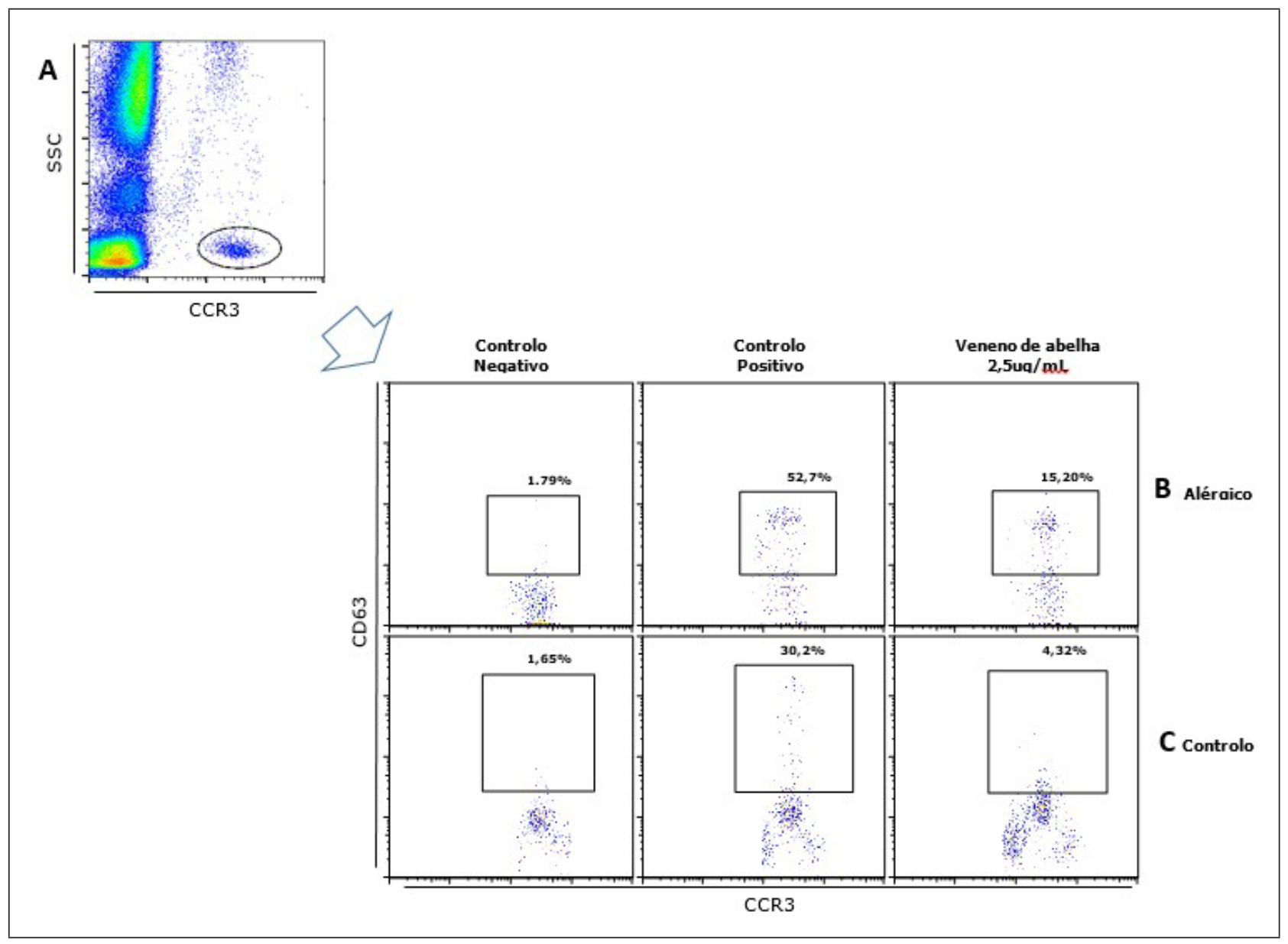

Figura I. Teste de ativação de basófilos no sangue total de um doente. A - Identificação de basófilos de acordo com a expressão dos níveis de CCR3. B - Expressão de CD63 em basófilos do doente, não estimulados (controlo negativo), estimulados com anti-FCERI (controlo positivo), estimulados com veneno de abelha (2,5 ug/ml); C - Expressão de CD63 em basófilos de controlo não estimulados (controlo negativo), estimulados com anti-FCERI (controlo positivo), estimulados com veneno de abelha (2,5 ug/ml) 
A avaliação da IgE específica para CCD demonstrou que 25 (38\%) dos 66 doentes polissensibilizados com IgE para extrato de veneno convencional tiveram lgE específica positiva para CCD (Quadro 2), correspondendo a 23 doentes que apresentaram reatividade cruzada entre espécies.

No grupo de doentes duplamente sensibilizados que não identificaram o inseto responsável, 7 (57\%) dos 12 doentes revelaram lgE esp para Api m I (5) ou Ves v I (2), enquanto os restantes 5 doentes tiveram IgE esp positiva para ambos os alergénios recombinantes. Curiosamente, 20 (16\%) doentes com história de anafilaxia apresentaram TC e IgE específica com extrato de veneno convencional negativos, assim como para os alergénios recombinantes testados.

Foi realizado o TAB com veneno de abelha e vespa em 5 doentes (uma vez que este é um método de diagnóstico dispendioso e moroso), todos com TC/IgE esp negativos, e 5 controlos, em 6 concentrações 0,0 I; 0,04; 0,2 , I, 2,5 e $5 \mu \mathrm{g} / \mathrm{ml}$. A população de basófilos foi identificada como $\mathrm{CCR}^{+}$. A ativação dos basófilos foi avaliada pela percentagem de expressão da molécula CD63 (Figura IA).

Três doentes tiveram resultado positivo com a concentração de $2,5 \mu \mathrm{g} / \mathrm{ml}$ com índice de estimulação superior a $2 \%$ e cut-off de ativação de basófilos de 10,57\%, $11,50 \%$ e $15,20 \%$, respetivamente, para veneno de $\mathrm{A}$ (Quadro 3). Os três doentes descreveram a última reação sistémica, após picada destes insetos, num período inferior a um ano. Em indivíduos não alérgicos a percentagem de ativação de basófilos foi inferior a 5\% (Figura IB e C).

\section{DISCUSSÃo}

Neste estudo, os autores avaliam a utilidade dos alergénios recombinantes no diagnóstico de alergia a veneno de himenópteros numa amostra de doentes da população portuguesa. Foram analisados os resultados de quantificação de anticorpos lgE esp para os componentes mole- culares major de veneno de himenópteros e os obtidos no doseamento com extrato total de veneno. Foi também avaliado o contributo do TAB no grupo de doentes com história sugestiva de alergia mas com TC e lgE esp negativos. Os resultados deste estudo com dados da população nacional são globalmente concordantes com os dados internacionais e apresentam implicações clínicas claras para abordagem destes doentes.

Foi demonstrado que a lgE esp para os componentes moleculares dos alergénios major de veneno de abelha e de vespa (fosfolipase A2-Api m I, veneno de espécies Vespula, fosfolipase AI-Ves v I, antigen5-Ves v 5 e Polistes, Pol d5), utilizados no diagnóstico de doentes com alergia a veneno de himenópteros, apresenta elevada sensibilidade e especificidade, uma vez que só um individuo do grupo-controlo apresentou positividade ao componente Ves $v 5$.

Sendo a imunoterapia (IT) com veneno de himenópteros considerada um tratamento altamente eficaz para estes doentes, prevenindo reações anafiláticas graves, a identificação do inseto responsável torna-se crucial, de forma a permitir uma seleção adequada da $I \mathrm{I}^{15-19}$. Embora, em alguns casos, esta possa ser feita pelo próprio doente, necessita de confirmação, através da realização de TC e quantificação de IgE específicas para os respetivos venenos ${ }^{16,17}$. Contudo, os TC podem revelar testes falsos-negativos, dada a baixa qualidade e concentração dos extratos alergénicos ${ }^{18}$. Por outro lado, o uso de concentrações mais elevadas pode resultar em reações falsamente positivas devido às propriedades tóxicas do veneno. Para além disso, mesmo doentes não sensibilizados podem apresentar testes falsos-positivos para extratos do veneno, o que pode ser explicado pela presença de determinantes dos carboidratos, possíveis responsáveis por reatividade cruzada, mas sem significado clínico ${ }^{17-21}$.

Cerca de $80 \%$ dos doentes com alergia a veneno de himenópteros apresentam resultados positivos nos TCe mais de $50 \%$ revelou anticorpos lgE esp para os venenos abelha, vespa e polistes, ainda que a maioria destes indi- 
víduos apenas tenha reagido à picada de um destes insetos. De acordo com a literatura, a dupla positividade baseada em TC positivos, com concentrações <l $\mu \mathrm{g} / \mathrm{ml}$, é observada em $40-50 \%$ dos doentes com alergia a himenópteros $^{17-19}$.

Torna-se então muito importante fazer a distinção entre verdadeira dupla sensibilização a ambos os venenos e reatividade cruzada. Neste contexto, é sugerido a avaliação de componentes moleculares para veneno de abeIha, vespa e polistes, já comercialmente disponíveis e considerados uma mais-valia. Pensou-se que, assim, o problema da identificação da dupla sensibilização/reatividade cruzada, em doentes com IgE específica positiva para CCD, ficaria solucionado. No entanto, como verificado no presente estudo, alguns destes indivíduos identificam IgE específica tanto para Api m I, Ves v I, Ves v 5 ou Pol d 5. Hofmann et al. concluíram que cerca de $50 \%$ dos doentes com dupla positividade nos TC e sensibilizados a CCD apresentavam IgE específica para ambos os marcadores Api m I e Ves v 5, o que vem colocar em causa a possibilidade de existência de reatividade cruzada. ${ }^{15}$ Neste estudo, $38 \%$ dos doentes polissensibilizados a veneno de abelha e veneno de vespa apresentaram positividade para CCD, sendo que só em $35 \%$ foi identificada reatividade cruzada.

Foi obtida uma sensibilidade mais baixa para o componente molecular Api m I e Ves v5 (68\% e 25\%, respetivamente) em relação à referida em outros estu$\operatorname{dos}^{18,20}$. sendo esta diferença causada provavelmente pela seleção da população de doentes. Em estudos anteriores, foram selecionados e analisados doentes com história de reação à picada de insetos nos últimos 12 meses, enquanto neste estudo foram incluídos e avaliados doentes com história documentada há mais de 10 anos, seguidos em consulta de alergia a himenópteros, fornecendo deste modo uma amostra mais realista da nossa prática clínica.

As diferentes sensibilidades obtidas para os componentes moleculares testados podem, de certa forma, refletir a possibilidade de alguns destes doentes poderem estar sensibilizados a outros componentes moleculares específicos de espécie e não testados neste estudo. Alargar o espetro de alergénios moleculares, como por exemplo, hialuronidase - Api m 2, fosfatase alcalina - Api m 3, melitina - Api m 4, icarapina - Api m 10, na avaliação do perfil de sensibilização destes doentes poderia representar uma mais valia, quer no diagnóstico, quer na escolha mais adequada de IT. ${ }^{22-24}$ É bem conhecido que a eficácia da IT depende de vários fatores, como duração do tratamento, dose de veneno durante a manutenção e tipo do veneno. A falha do tratamento mais frequente é com veneno de abelha, tendo sido recentemente referida a falta da proteína Api m 3 e Api m 10 na composição da vacina ${ }^{22}$.

A utilidade do TAB no diagnóstico da alergia a veneno de himenópteros tem sido demonstrada em diversos trabalhos, principalmente no sentido da avaliação de dupla sensibilização/reatividade cruzada. Neste contexto, Balzer et al. referem que o TAB realizado com alergénios recombinantes pode incrementar a especificidade, representando assim um importante e confiável teste de diagnóstico in vitro em doentes alérgicos a himenópteros ${ }^{18,21}$. Também no sentido de melhorar a sua sensibilidade têm sido propostas algumas sugestões, quer no que diz respeito à identificação da população de basófilos, quer na avaliação da ativação, com utilização de novas moléculas (marcadores de ativação, CD63 ou CD203c, e de identificação de basófilos-CCR3, HLA-DR, CDI23) ${ }^{24,25}$. No nosso estudo foi feita a identificação da população de basófilos com a utilização da molécula CCR3, proposta mais recentemente. Em relação aos doentes em que foi realizado TAB, apresentavam TC e IgE esp negativas para ambos os venenos e o teste foi positivo apenas para o veneno de abelha em 3 dos casos do grupo de estudo, permitindo a identificação do inseto e a iniciação de IT com este veneno. Estes 3 doentes referiam ter tido sido picados num período inferior a um ano. Como referido na literatura, o tempo decorrido entre a reação e a realização do teste é considerado um fator relevante nesta avaliação ${ }^{23,25}$. Uma vez que estes doentes tinham uma história de RS grave, com TC e IgE 
esp negativos, sendo difícil a tomada de decisão, foi uma mais-valia a avaliação pelo TAB. No entanto, não podemos descartar a hipótese, como referido na literatura, da relevância da realização do TAB com alergénios recombinantes $^{18,24,25}$.

Em conclusão, a análise do perfil de sensibilização utilizando um painel de componentes moleculares para veneno de abelha, vespa e Polistes, aumenta a sensibilidade e precisão do diagnóstico em doentes com alergia a veneno de himenópteros. Além disso, contribui para a discriminação entre a verdadeira dupla sensibilização e reatividade cruzada em doentes com resultados duplamente positivos para extratos convencionais do veneno. Este facto permite otimizar a seleção de doentes para imunoterapia, evitando protocolos de tratamento desnecessários com ambos os venenos. $O$ TAB poderá ser considerado útil em casos difíceis e de diagnóstico inconclusivo, mas mais estudos e com maior número de doentes deverão ser realizados.

\section{DECLARAÇÕES ÉTICAS}

Confidencialidade dos dados. Os autores declaram que seguiram os protocolos sobre a publicação dos dados individuais e que todos os doentes incluídos no estudo receberam informações claras e completas, dando o seu consentimento informado por escrito para participação.

\section{Direito à privacidade e consentimento infor-} mado. Os autores obtiveram o consentimento informado de todos os doentes e indivíduos de grupo-controlo. autor para correspondência possui este documento.

\section{Proteção de seres humanos em pesquisa. Os} autores declaram que os procedimentos seguidos estavam de acordo com os regulamentos da Comissão de Ética em Pesquisa Clínica e de acordo com os da Associação Médica Mundial e a Declaração de Helsínquia.

\section{Apoio financeiro}

Os autores agradecem à Thermofisher Diagnostics o apoio pela cedência de alergénios recombinantes utilizados no estudo.

\section{Contributo dos autores}

LP, EP, MCPS, escrita do projeto; LP, EP, AM, MCPS desenvolveram o estudo e a análise de dados; LP, EP e MPB estiveram envolvidos na investigação clínica; LP, EP, MPB e MCPS, discussão de resultados; LP e MCPS, escrita do artigo.

\section{Conflito de interesse}

Os autores declaram que não apresentam quaisquer conflitos de interesse.

Contacto:

Letícia Pestana

E-mail: marialeticiapestana@gmail.com

\section{REFERÊNCIAS}

I. Mittermann I, Zidarn M, Silar M, Markovic-Housley Z, Aberer W, Korosec P, et al. Recombinant allergen-based IgE testing to distinguish bee and wasp allergy. J Allergy Clin Immunol 2010;125: 1300-7.

2. Bilo MB, Rueff F, Mosbech H, Bonifazi F, Oude-Elberink JNG. EACCI Interest Group on Insect Venom Hypersensitivity. Diagnosis of hymenoptera venom allergy. Allergy 2005;60:1339-49.

3. Schiener M, Eberlein B, Moreno-Aguilar C, Pietsch G, Serrano $P$, Mclntyre M, et al. Application of recombinant antigen 5 allergens from seven allergy-relevant Hymenoptera species in diagnostics. Allergy 2017; 72:98-108.

4. Sastre J, Sastre-lbañez M. Molecular diagnosis and immunotherapy. Curr Opin Allergy Clin Immunol 2016;16:565-70.

5. Mahler V, Gutgesell C, Valenta R, Fuchs T. Natural rubber latex and hymenoptera venoms share immunoglobin $E$ epitopes accounting for cross-reactive carbohydrate determinants. Clin Exp Allergy 2006; 36:1446-56.

6. Michel J, Brockow K, Darsow U, Ring J, Schmidt-Weber CB, Grunwald $T$, et al. Added sensitivity of component-resolved diagnosis in hymenoptera venom-allergic patients with elevated serum tryptase and/or mastocytosis. Allergy 2016;71:65I-60. 
7. Vachová M, Panzner P, Malkusová I, Hanzlíková J, Vlas T. Utility of laboratory testing for the diagnosis of Hymenoptera venom allergy. Allergy Asthma Proc 2016;37:248-55.

8. Matricardi PM, Kleine-Tebbe J, Hoffmann HJ, Valenta R, Hilger C, Hofmaier S, et al. EAACI Molecular Allergology User's Guide. Pediatr Allergy Immunol 2016;27 Suppl 23:I-250.

9. Selb J, Kogovšek R, Šilar M, Kosnik M, Korošec P. Improved recombinant Api $\mathrm{m} \mathrm{I-}$ and Ves $v 5$-based IgE testing to dissect bee and yellow jacket allergy and their correlation with the severity of the sting reaction. Clin Exp Allergy 2016;46:62I-30.

10. Savi E, Peveri S, Makri E, Pravettoni V, Incorvaia C. Comparing the ability of molecular diagnosis and CAP-inhibition in identifying the really causative venom in patients with positive tests to Vespula and Polistes species. Clin Mol Allergy 2016;14:3.

II. Muller UR. Insect sting allergy. Clinical picture, diagnosis and treatment. Stuttgart; New York: Gustav Fischer, 1990.

12. Scherer K, Weber JM, Jermann TM, Krautheim A, Tas E, Ueberschlag EV, et al. Cellular in vitro assays in the diagnosis of Hymenoptera venom allergy. Int Arch Allergy Immunol 2008; 146:122-32.

13. Caruso B, Bonadonna P, Bovo C, Melloni N, Lombardo C, Senna $\mathrm{G}$, et al. Wasp venom allergy screening with recombinant allergen testing. Diagnostic performance of rPol d 5 and rVes $v 5$ for differentiating sensitization to Vespula and Polistes subspecies. Clin Chim Acta.2016; 30:170-3.

14. Mertens M, Amler S, Moerschbacher BM, Brehler R. Cross-reactive carbohydrate determinant strongly affect the results of the basophil activation test in Hymenoptera-venom allergy. Clin Exp Allergy 2010; 40:1333-45.

15. Hoffmann HJ, Santos AF, Mayorga C, Nopp A, Eberlein B, Ferrer M. et al. The clinical utility of basophil activation testing in diagnosis and monitoring of allergic disease. Allergy 2015;70:1393-405.

16. Galindo-Bonilla PA, Galán-Nieto A, Alfaya-Arias T, García-Rodríguez C, de la Roca-Pinzón F. Component-resolved diagnosis in vespid venom-allergic individuals. Allergol Immunopathol (Madr) 2015;43:398-402.

17. Van Gasse AL, Mangodt EA, Faber M, Sabato V, Bridts CH, Ebo DG. Molecular allergy diagnosis: status anno 2015. Clin Chim Acta 2015;15:54-6I.

18. Balzer L, Pennino D, Blank S, Seismann H, Darsow U, Schnedler Met al. Basophil activation test using recombinant allergens: highly specific diagnostic method complementing routine tests in wasp venom allergy. PLoS One 2014:17;9:e108619.

19. Golden $\mathrm{DB}, \mathrm{New}$ directions in diagnostic evaluation of insect allergy. Curr Opin Allergy Clin Immunol 2014;14:334-9.

20. Köhler J, Blank S, Müller S, Bantleon F, Frick M, Huss-Marp J. et al. Component resolution reveals additional major allergens in patients with honeybee venom allergy. J Allergy Clin Immunol 2014;133:1383-9, el-6.

21. Ebo DG, Van Vaerenbergh M, de Graaf DC, Bridts CH, De Clerck LS, Sabato V. In vitro diagnosis of Hymenoptera venom allergy and further development of component resolved diagnostics. Expert Rev Clin Immunol 2014;10:375-84.

22. Spillner E, Jakob T. Predominant Api m I0 sensitization as risk factor for treatment failure in honey bee venom immunotherapy. J Allergy Clin Immunol 2016; 138:1663-7I.

23. Ollert M, Blank S. Anaphylaxis to Insect Venom Allergens: Role of Molecular Diagnostics. Curr Allergy Asthma Rep 2015; 15: 26 9-II.

24. Korosec P, Erzen R, Silar M, Bajrovic N, Kopac P, Kosnik M. Basophil responsiveness in patients with insect sting allergies and negative venom specific immunoglobulin $E$ and skin prick test results. Clin Exp Allergy 2009; 39: 1730-7.

25. Jakob T, Rafei-Shamsabadi D, Spillner E, Müller S. Diagnostics in Hymenoptera venom allergy: current concepts and developments with special focus on molecular allergy diagnostics. Allergo J Int 2017; 26:93-105. 